\title{
AN ENERGY-BASED METHOD FOR NUMERICAL FATIGUE ANALYSIS OF MULTIDIRECTIONAL CARBON FIBRE REINFORCED PLASTICS
}

\author{
P. Wagner ${ }^{\text {* }}$, T. Bruder ${ }^{\mathrm{a}}$, W. Van Paepegem ${ }^{\mathrm{b}}$, M. May ${ }^{\mathrm{c}}$ \\ ${ }^{a}$ BMW Group, Forschungs- und Innovationszentrum, Knorrstr. 147, 80788 Munich, Germany \\ ${ }^{b}$ Ghent University, Department of Materials Science and Engineering, Technologiepark-Zwijnaarde \\ 903, 9052 Zwijnaarde, Belgium \\ ${ }^{c}$ Fraunhofer Institute for High-Speed Dynamics, Ernst-Mach-Institut, EMI, Eckerstr. 4, 79104 \\ Freiburg, Germany \\ *Peter.Wagner@bmw.de
}

Keywords: CFRP, Fatigue, Numerical Analysis.

\begin{abstract}
This paper describes experiments on multi-axial fibre reinforced plastic laminates, which were performed to obtain calibration data for numerical fatigue analyses. For this purpose, fatigue tests of laminates with multi-directional layers subjected to constant amplitude and block loading $(0 \leq \mathrm{R}<1$ or $\mathrm{R}>1)$ are analysed. The presented simulation results display the fatigue behaviour of carbon fibre reinforced plastics (CFRP) for unidirectional loading conditions and a selected laminate.
\end{abstract}

\section{Introduction}

Current research and development activities in the automotive industry focus on fuel efficiency and sustainable production. Both governmental regulations and increased customer demand regarding sustainability of products have led to greater efforts to fulfil these demands. To improve structural design, it is essential to utilise new materials such as carbon fibre reinforced plastics (CFRP) which combine high specific strength, stiffness as well as energy absorption (crash) with an overall low material density (weight).

The aviation industry is already utilising composite materials such as CFRP with great success (see, e.g. Airbus A350 or Boeing 787). However, modelling of fatigue behaviour has long been neglected by the aerospace industry due to safety regulations requiring design based on extremely low maximum strain allowables which in turn result in low susceptibility to fatigue damage. New circumstances, automotive industry interests in large-scale production, recent progress in large scale manufacturing technology (high pressure resin transfer moulding - HPRTM), forced the topic of fatigue-induced failure into focus. Today, CFRPs are designed and calculated mainly for static or crash loads. In order to address the problem of fatigue the components are designed using a high safety factor. The forces used to design the component are much higher than the regular forces which might be seen during the service life. 
The literature suggests that although several types of models such as fatigue life models, phenomenological strength and stiffness models and progressive damage models to simulate the quasi-static and fatigue behaviour of carbon fibre reinforced plastics have been proposed there is no model tailored to meeting the current demands of the automotive industry. Most models described are not able to predict the degradation of the material or in-service misuse or damage sustained before or during testing/usage (e.g. impact damage), which is of importance when designing CFRP components. Additionally there is no simulation tool available which is able to calculate entire automotive structures in a reasonable timescale [1 4].

This paper presents an energy-based method on laminate level for the numerical fatigue analysis of CFRP structures. The model is derived from quasi-static and from constant amplitude tests on coupon specimens. The model is used to simulate the stiffness degradation of coupon specimens exposed to variable amplitude loadings with load ratios $0 \leq \mathrm{R}<1$ or $R>1$, respectively. The load ratio is defined using the upper and lower load $\sigma_{U P}$ and $\sigma_{\text {LO }}$ which is applied to the specimen.

$R=\frac{\sigma_{L O}}{\sigma_{U P}}$

The method enables the analysis of the stiffness degradation of the tested samples and is aimed at calculating the stiffness degradation of components.

\section{Materials and testing procedure}

The CFRP samples were produced using a high pressure resin transfer moulding process. The samples were physically and chemically analysed (density, glass transition temperature, differential scanning calorimetry, thermography and ultrasonic testing) to guarantee the uniformity of all samples. The CFRP plates were water jet cut into $250 \times 25 \times 2.1 \mathrm{~mm}^{3}$ coupons for tensile testing and $150 \times 25 \times 2.1 \mathrm{~mm}^{3}$ for compression testing. Glass tabs were applied to the ends of the specimens to achieve a better load distribution. The multidirectional lamina has a layup of $[+45 /-45 / 0]_{\mathrm{s}}$. The main fibre direction is defined as $\mathrm{x}$-direction while the direction normal to the fibres is defined as y-direction. All experiments and results referred to in this paper are of samples tested in y-direction. Experiments were also conducted in $\mathrm{x}$ - direction. However, the scope of this paper is on matrix dominated failure modes rather than fiber failure modes. Therefore these results are not shown in this paper.

\subsection{Quasi-static experiments}

For quasi-static experiments following standards were used:

- DIN EN ISO $527 \quad$ Quasi-static tension and

- DIN EN ISO 14126 Quasi-static compression

The quasi-static tension experiments, for the multidirectional laminates in 90 degree direction were conducted using a universal test machine TIRAtest 2300 according to standard ISO 7500-1 with a load cell capacity of $10 \mathrm{kN}$. The measurement was conducted using a monochromatic video-extensometer ME46 of the Messphysik Laborgerätegesellschaft mbH with the lowest resolution of $4 \mu \mathrm{m}$ and the highest resolution of $0.1 \mu \mathrm{m}$.

The quasi-static compression experiments were conducted using a universal test machine HP250 system in accordance to standard ISO 7500-1. A loading cell with $10 \mathrm{kN}$ 
capacity was used. The required data was recorded using two strain gauges 6/120ZE LY21 S3-4-1M with a grid length of $6 \mathrm{~mm}$ of Hottinger Baldwin Messtechnik GmbH (HBM) in addition to a measurement amplifier.

\subsection{Constant amplitude loading tests}

The tests under constant amplitude loadings were conducted using Schenk servo hydraulic testing machines with nominal forces of 25 to $50 \mathrm{kN}$. The force was measured with a load cell GTM series K Nr.46678. The peak data points of the applied force and the maximum strain were used to calculate, the stiffness degradation of the specimen. Constant amplitude fatigue tests on coupon specimens were performed under load control at six load levels and a load ratio of $R=0$ with a constant frequency of $f=10 \mathrm{~Hz}$. All specimens were tested until rupture occurred or until 2.000 .000 cycles were reached.

Constant amplitude compression data was obtained using the following procedure. The tests were performed using anti-buckling guides to conduct accurate measurements and prevent the specimen from buckling. Two clip gauges, MTS 632.11F-20 and MTS 632.03F-30, were attached to the front and back side of the specimen ensuring an overlap of the measurement range as shown in figure 1. Furthermore tests with a compression mean load were performed with load conditions of $\mathrm{R}=10$ and a constant frequency of $\mathrm{f}=4 \mathrm{~Hz}$. A servo hydraulic rig with a GTM load cell of $100 \mathrm{kN}$ and a measurement range of $60 \mathrm{kN}$ in accordance with standard ISO 7500-1 was used. Figure 1 shows the setup of the clip gauges.

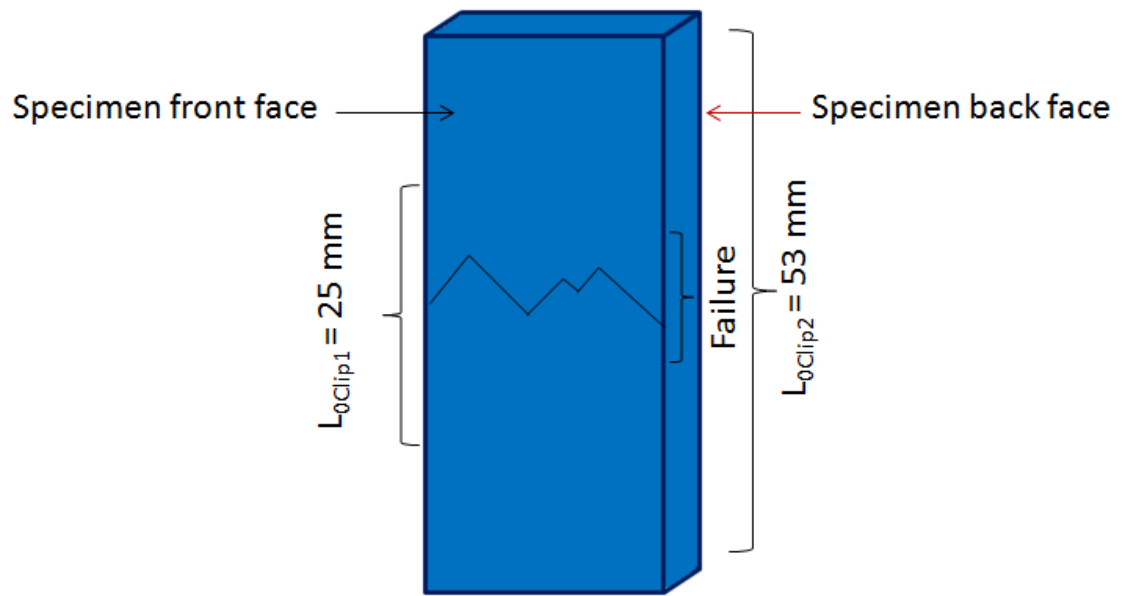

Figure 1. Schematic mounting of the extension meters for tension compression loading blocks. One extensometer is mounted on the front face and another extensometer is mounted on the back face of the specimen to ensure a stable record of the experimental data.

\subsection{Tension and compression load blocks}

The tension-tension loading blocks were tested using a servo-hydraulic testing rig at the Materialforschung und Anwendungstechnik GmbH in Dresden (IMA) with a load cell of $25 \mathrm{kN}$ and a measurement range of $60 \mathrm{kN}$ in accordance with standard ISO7500-1 using the MTS Flex Test SE controller and MTS Clip-Gage 632.11F-20. The tension-compression loading block experiments were conducted using a servo hydraulic rig at IMA with a GTM load cell of $100 \mathrm{kN}$ and a measurement range of $60 \mathrm{kN}$ in accordance with standard ISO 7500-1. Two clip-gauges (MTS 632.11F-20 and MTS 632.03F-30) were applied to the specimen. Because there are no standardized instructions a specimen size of $150 \times 25 \times 2.1 \mathrm{~mm}^{3}$ was chosen. This is similar to the test programm for pure compressive loading. The tests were performed using anti-buckling guides to conduct to prevent buckling 
of the specimen. The clip gauges were attached according to figure 1. Furthermore tests with a tensile mean load were performed with load ratios of $\mathrm{R}=0.1, \mathrm{R}=0.5$ and $\mathrm{R}=0.9$ and a constant frequency of $\mathrm{f}=4 \mathrm{~Hz}$. This low frequency was chosen to avoid frictional heating of the specimen. All samples were tested until at least four loading blocks of the loading block spectrum were completed. The loads and ratios applied can be seen in table 1 to table 4 .

\begin{tabular}{|l|c|c|c|}
\hline \multicolumn{4}{|c|}{ Tensile loading blocks (TTTT) } \\
\hline Block & ouP in \% to $\mathrm{R}_{\mathrm{m}}$ & $\mathrm{R}$ & Number of cycles \\
\hline 1 & 51 & 0.1 & 5000 \\
\hline 2 & 42 & 0.1 & 50000 \\
\hline 3 & 48 & 0.1 & 10000 \\
\hline 4 & 41 & 0.1 & 60000 \\
\hline
\end{tabular}

Table 1: Test sequence with 4 pulsating tension loading blocks with constant $\mathrm{R}$ value.

\begin{tabular}{|l|c|c|c|}
\hline \multicolumn{4}{|c|}{ Tensile loading blocks (TTTT) } \\
\hline Block & $\sigma_{\text {UP }}$ in $\%$ to $\mathrm{R}_{\mathrm{m}}$ & $\mathrm{R}$ & Number of cycles \\
\hline 1 & 45 & 0.1 & 25000 \\
\hline 2 & 45 & 0.5 & 25000 \\
\hline 3 & 45 & 0.1 & 25000 \\
\hline 4 & 45 & 0.9 & 25000 \\
\hline
\end{tabular}

Table 2: Test sequence with 4 cyclic tension loading blocks with upper load level $\sigma$ UP and variable $\mathrm{R}$ value.

\begin{tabular}{|l|c|c|c|}
\hline \multicolumn{4}{|c|}{ Tensile loading blocks (TTTT) } \\
\hline Block & ouP in \% to $\mathrm{R}_{\mathrm{m}}$ & $\mathrm{R}$ & Number of cycles \\
\hline 1 & 45 & 0.1 & 25000 \\
\hline 2 & 45 & 0.9 & 25000 \\
\hline 3 & 45 & 0.1 & 25000 \\
\hline 4 & 45 & 0.5 & 25000 \\
\hline
\end{tabular}

Table 3: Test sequence with 4 cyclic tension loading blocks with upper load level $\sigma$ UP and variable $\mathrm{R}$ value.

\begin{tabular}{|l|c|c|c|}
\hline \multicolumn{4}{|c|}{ Tensile Compression loading blocks (TTCT) } \\
\hline Block & $\sigma$ in \% to $\mathrm{R}_{\mathrm{m}}$ & $\mathrm{R}$ & Number of cycles \\
\hline 1 & $\sigma_{\text {UP: }} 48$ & 0.1 & 10000 \\
\hline 2 & $\sigma_{\text {UP: }} 42$ & 0.1 & 50000 \\
\hline 3 & $\sigma_{\text {LO }} 56$ & 10 & 20000 \\
\hline 4 & $\sigma_{\text {UP }}: 51$ & 0.1 & 5000 \\
\hline
\end{tabular}

Table 4: Load blocks for cyclic tension - compression experiments. A single compression load block is included in the load spectrum.

\begin{tabular}{|l|c|c|c|}
\hline \multicolumn{4}{|c|}{ Compression Tensile loading blocks (CCTC) } \\
\hline Block & $\sigma$ in $\%$ to $\mathrm{R}_{\mathrm{m}}$ & $\mathrm{R}$ & Number of cycles \\
\hline 1 & $\sigma_{\text {LO }} 56$ & 10 & 20000 \\
\hline 2 & $\sigma_{\text {LO }} 54$ & 10 & 40000 \\
\hline 3 & $\sigma_{\text {UP: }} 46$ & 0.1 & 15000 \\
\hline 4 & $\sigma_{\text {LO }}: 58$ & 10 & 10000 \\
\hline
\end{tabular}

Table 5: Load blocks for cyclic compression - tension experiments. A single tension load block is included in the load spectrum. 


\section{Proposed model for fatigue simulation of carbon fibre reinforced plastics}

The fatigue behaviour of CFRPs depends on the stacking sequence of the layers, the production process (HPRTM, pre-preg) used, applied loading, the direction of the applied loading, the load ratio $\mathrm{R}$ as well as the environmental conditions. Due to the various influences and the complex damage behaviour of the material $[5,6]$ a multitude of fatigue models have been developed. A comprehensive review of fatigue models for composite materials is given in [3]. They can be classified as classic fatigue models, phenomenological residual strength, stiffness models and progressive damage models.

Here, a model originally developed for modelling quasi-static damage in composite materials [7] was extended to describe the fatigue behaviour of the material [8]. The advantage of using the mesoscale modelling level results in the decrease of computational resources which can be enormous, depending on the calculated structure. In the case of plane stress assumption, the total strain energy for the damaged material at the meso-level can be formulated, as follows [9]:

$$
E_{D}=\frac{1}{2} \cdot\left[\begin{array}{l}
\frac{\left\langle\sigma_{11}\right\rangle_{+}^{2}}{E_{11}^{0}}+\frac{\varphi\left\langle\sigma_{11}\right\rangle_{-}^{2}}{E_{11}^{0}}-\left(\frac{v_{12}^{0}}{E_{11}^{0}}+\frac{v_{21}^{0}}{E_{22}^{0}}\right) \cdot \sigma_{11} \cdot \sigma_{22}+ \\
+\frac{\left\langle\sigma_{22}\right\rangle_{+}^{2}}{\left(1-d^{\prime}\right) \cdot E_{22}^{0}}+\frac{\left\langle\sigma_{22}\right\rangle_{-}^{2}}{\left(1-d^{\prime}\right) \cdot E_{22}^{0}}+\frac{\sigma_{12}^{2}}{(1-d) \cdot G_{12}^{0}}
\end{array}\right]
$$

where 11,22 and 12 are the fibre, transverse and shear direction, $\mathrm{d}$ and $\mathrm{d}$ ' are two scalar internal variables which are constant within the thickness of the ply and $\langle\cdot\rangle_{+}$denotes the positive part and $\langle\cdot\rangle_{-}$the negative. $\varphi$ is a material function which takes into account the nonlinear response in compression. E denotes the Youngs modulus and $\mathrm{G}$ the shear modulus, $\sigma$ the applied stress and $v$ the poisson ratio. The first model distinguishes two ply degradation mechanisms that contribute to damage development, matrix microcracking and fibre matrix debonding. The advantage of using the total strain energy model is based on the fact that the potential energy is the same between a meso and a micro model, which enables the simulation of the material in a larger scale factor and therefore safe computational time. Further model developments for quasi-static and high rate problems were later proposed, more detailed information can be found in [10 - 14].

\subsection{Model description}

The simulation method is based on the total strain energy and therefore makes it possible to calculate the damage propagation similar to the crack propagation described by the Paris Law [15]. Therefore it is necessary to have the ability to characterize quasi-static and fatigue damage.

For the calculation the total strain energy of a plate at laminate level $\left(\mathrm{E}_{\mathrm{D}}\right)$ is used. Various damage variables corresponding to the load direction are further included $\left(d_{x x}, d_{y y}, d_{x y}\right)$ to 
describe the degradation behaviour of the material. The total strain energy for a cycle ( $\left.E_{D L W}\right)$ follows as:

$$
E_{D L W}^{i}=\frac{1}{2} \cdot\left[\begin{array}{l}
\frac{\sigma_{x x}^{2}}{S_{x x}^{j} \cdot\left(1-d_{x x T}^{i}\right)}+\frac{\sigma_{-x x}^{2}}{S_{x x}^{j} \cdot\left(1-d_{x x C}^{i}\right)}-\frac{v_{x y}}{S_{x x}^{j} \cdot\left(1-d_{x x T}^{i}\right)} \sigma_{x x} \sigma_{y y}+ \\
+\frac{\sigma_{y y}^{2}}{S_{y y}^{j} \cdot\left(1-d_{y y T}^{i}\right)}+\frac{\sigma_{-y y}^{2}}{S_{y y}^{j} \cdot \beta \cdot\left(1-d_{y y C}^{i}\right)}-\frac{v_{y x}}{S_{y y}^{j} \cdot\left(1-d_{y y T}^{i}\right)} \sigma_{x x} \sigma_{y y}+ \\
+\frac{\sigma_{x y}^{2}}{G_{x y}^{j}\left(1-d_{x y}^{i}\right)}
\end{array}\right]
$$

where $\mathrm{xx}$, yy and xy are respectively the components in loading direction, transverse loading direction and shear, $\mathrm{d}_{\mathrm{xy}}^{\mathrm{i}}$ are the defined damage variables in $\mathrm{xy}$ direction for the number of cycles $\mathrm{i}$ which are constant within the thickness of the ply. $S_{x x}^{j}$, denotes the last calculated stiffness before the cycle step i. T and $\mathrm{C}$ denote the damage for tension or compression. To calculate the free damage energy release rate of the system according to the applied load the derivative of $\mathrm{E}_{\mathrm{DLW}}$ is required in load direction such as $\mathrm{d}_{\mathrm{xy}}^{\mathrm{i}}$

$$
Y_{d_{x x}}^{i}=\frac{\partial E_{D L W}^{i}}{\partial d_{x x T}^{i}}=\frac{1}{2} \cdot\left\{\frac{\sigma_{x x T}^{2}}{\left(1-d_{x x T}^{i}\right)^{2} \cdot E_{x x}^{i}}-\frac{v_{x y}^{0}}{\left(1-d_{x x T}^{i}\right)^{2} \cdot E_{x x}^{i}} \sigma_{x x T} \cdot \sigma_{y y T}\right\}
$$

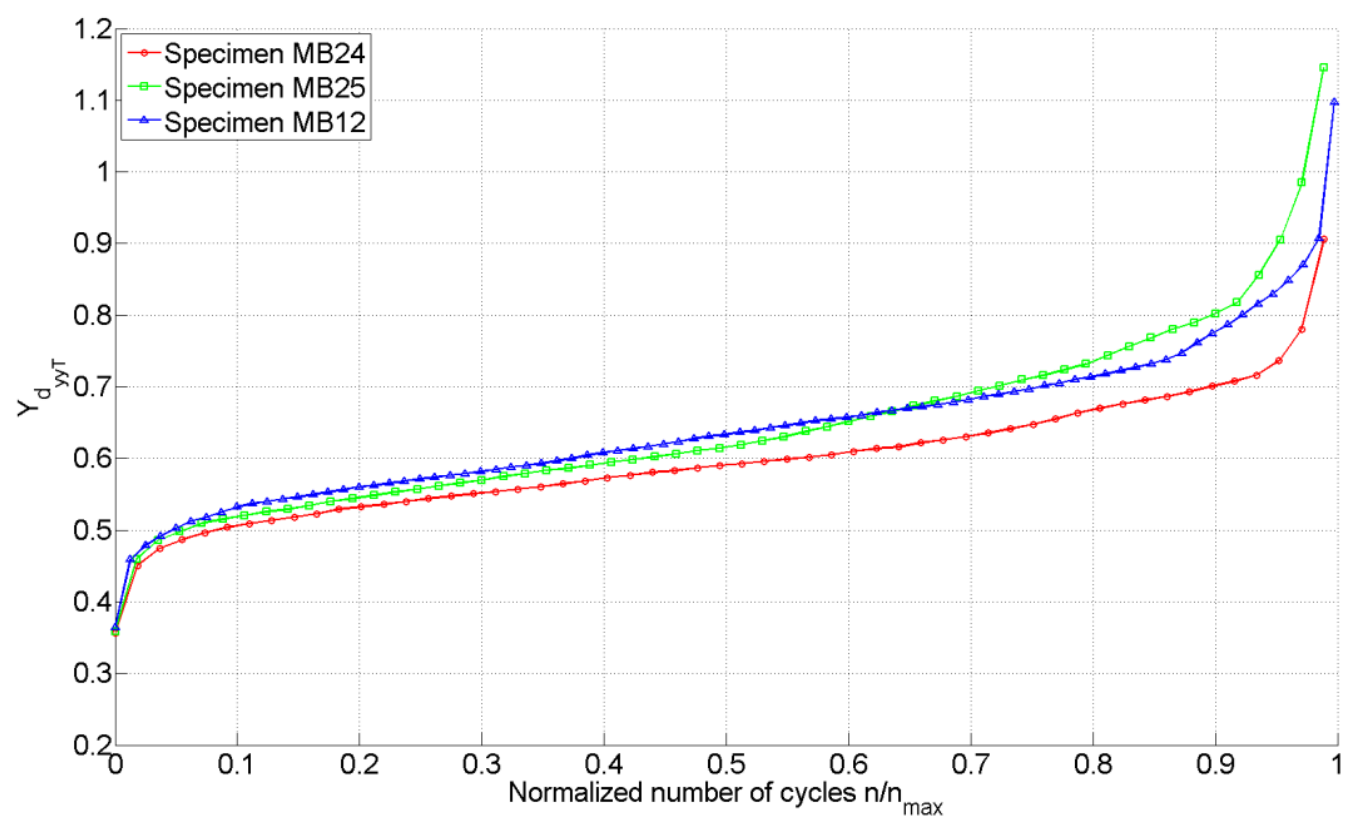

Figure 2: Free damage energy release rate. The $\mathrm{x}$-axis is defined as the normalized number of cycles $\mathrm{n} / \mathrm{n}_{\max }$.

Figure 2 shows the calculated free damage energy release rate $Y_{d_{x x}}^{i}=\frac{\partial E_{D L W}^{i}}{\partial d_{x x T}^{i}}$ for three specimens subjected to constant amplitude loading experiments. While the free damage energy release rate is quite attractive to plot and does point to similarities of the Paris Law it is very impractical for numerical simulation. Therefore equation 2 needs to be broken down further. In order to describe the damage evolution based on the number of applied cycles $n$ it 
is necessary to formulate the material behaviour into a function. Using the theorem developed by Van Paepegem [16] for tension: 
$\frac{\partial d}{\partial n}=c_{1} \cdot \sum(\sigma, d) \cdot e^{\left(-c_{2} \cdot \frac{d}{\sqrt{\sum(\sigma, d)}}\right)}+c_{3} \cdot D \cdot \sum(\sigma, d)^{2} \cdot\left[1+e^{\left(c_{5} \cdot\left(\sum(\sigma, d)-c_{4}\right)\right)}\right]$

where $c_{1}$ to $c_{5}$ are material constants and $\sum(\sigma, d)$ is the fatigue failure index, it is possible to propose a function which describes the evolution of the damage variable depending on number of cycles $n$ and the stress amplitude $\sigma_{\mathrm{a}}$.

For calculation purposes it is necessary to normalise $n, \sigma_{a}$ and $d$ to receive a normalised

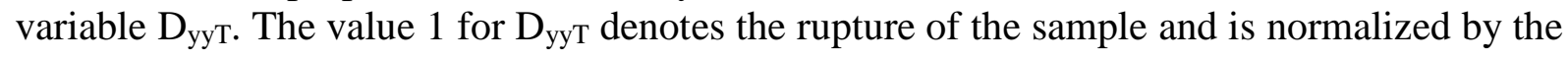
maximum value of $d_{x x}^{n_{\max }}$ or the damage according to load direction. The normalization of the damage value was necessary to calculate the material parameters. It further has the advantage to provide a better understanding of the state of damage in the material.

$$
\mathrm{D}_{\mathrm{yyT}}=\mathrm{a} \cdot\left(\tilde{\sigma}_{y y T} \cdot \tilde{\mathrm{n}}_{\mathrm{yyT}}\right) \cdot \mathrm{e}^{\left((-1) \cdot \mathrm{b} \cdot \sqrt{\tilde{\sigma}_{y y T} \cdot \tilde{\mathrm{n}}_{\mathrm{yyT}}}\right)}+\mathrm{c} \cdot\left(\tilde{\sigma}_{y y T} \cdot \tilde{\mathrm{n}}_{\mathrm{yyT}}\right)^{f} \cdot\left(1+\mathrm{e}^{\left(\mathrm{g} \cdot\left(\left(\tilde{\sigma}_{y y T} \cdot \tilde{\mathrm{n}}_{\mathrm{yyT}}\right)-h\right)\right)}\right)
$$

The normalised damage propagation $\mathrm{D}$ is calculated for each load applied in the constant amplitude loading tests. The variables a, b, c, g, h are material constants which are calculated using the results of several constant amplitude loading tests. Those variables are then further linearly approximated as a function of $\sigma$ for all possible applied loads to achieve a consistent material characterization. $\tilde{\sigma}_{y y T}$ denotes the normalized stress amplitude using the maximum achieved stress before failure $\sigma_{f}$ while $\tilde{\mathrm{n}}_{\mathrm{yyT}}$ denotes the normalized number of cycles for each load level. The maximum number of cycles $n_{y y T_{\max }}$ until failure or rupture of the specimens occurs is calculated using the well used and known statistical approach of the S-N curves (fig. $3)$.

$$
\begin{gathered}
\tilde{\sigma}_{y y T}=\frac{\sigma_{y y T}}{\sigma_{f}} \\
\tilde{\mathrm{n}}_{\mathrm{yyT}}=\frac{n_{y y T}}{n_{y y T_{\max }}}
\end{gathered}
$$

Having formulated the damage calculation and the damage energy release rate equation it is possible to calculate the effective free damage energy release rate $\mathrm{Y}_{\text {eff }}$ of the upper and lower load $\sigma_{U P}$ and $\sigma_{\mathrm{LO}}$ which is applied to the specimen when conducting an experiment with a load ratio within of $0<\mathrm{R}<1$ or $\mathrm{R}>1$, respectively. Using this data (fig. 4) it is possible to calculate the effective stress $\sigma_{\text {eff }}$ and therefore the damage sustained by the specimen exposed to loading with different amplitudes loadings.

$$
Y_{e f f_{y y T}}\left(\sigma_{e f f_{y y T}}\right)=Y_{d_{y y T}}^{i}\left(\sigma_{U P_{y y T}}\right)-Y_{d_{y y T}}^{i}\left(\sigma_{L O_{y y T}}\right)
$$

To end the simulation following exit criteria were defined:

I. D reaches the value defined for rupture.

II. The maximum level of the damage energy release rate for the applied load is reached.

III. The difference of the damage energy release rate between two cycles is higher than the allowed energy density release rate for quasi-static loading. 


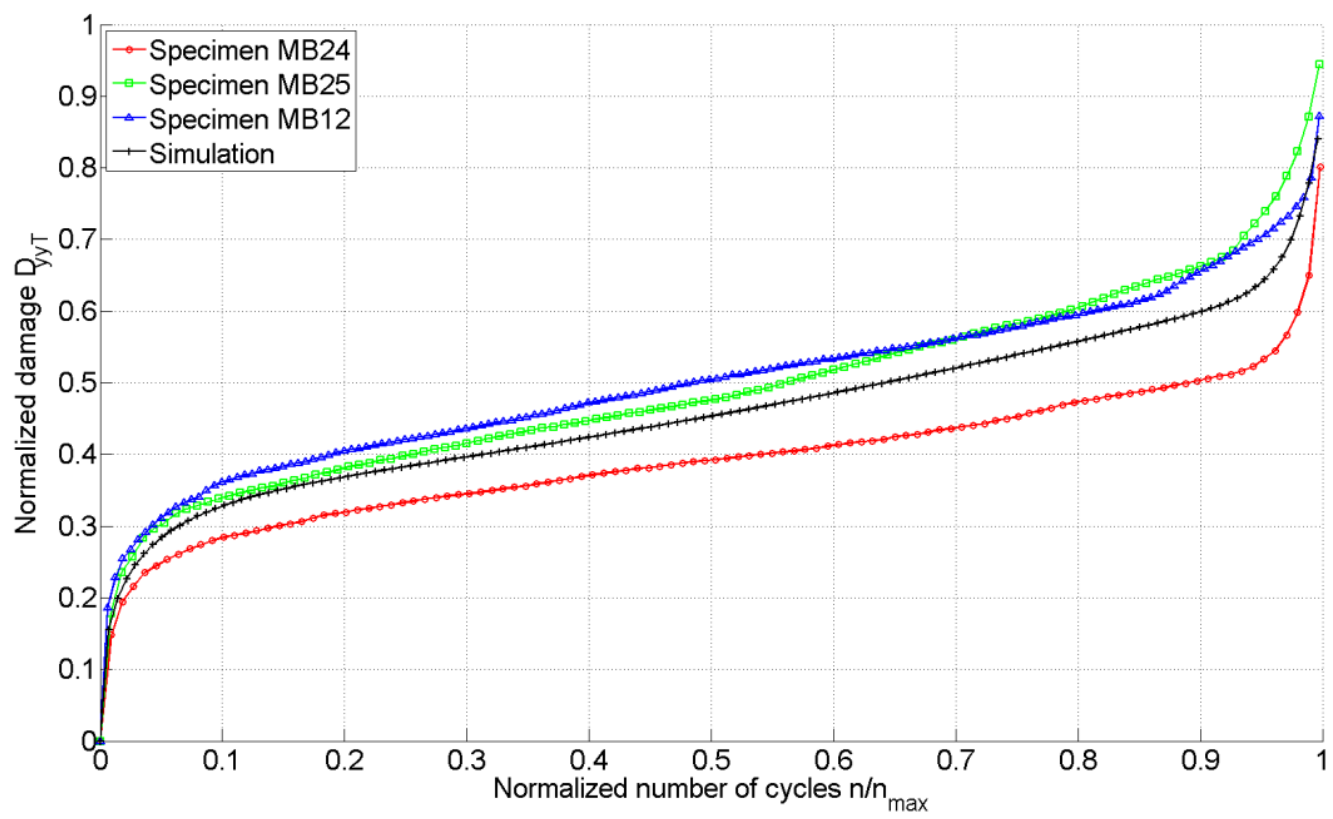

Figure 3: Damage propagation for a selected multidirectional laminate. Displayed are experimentally obtained data of constant amplitude loading tests and the approximated simulation.

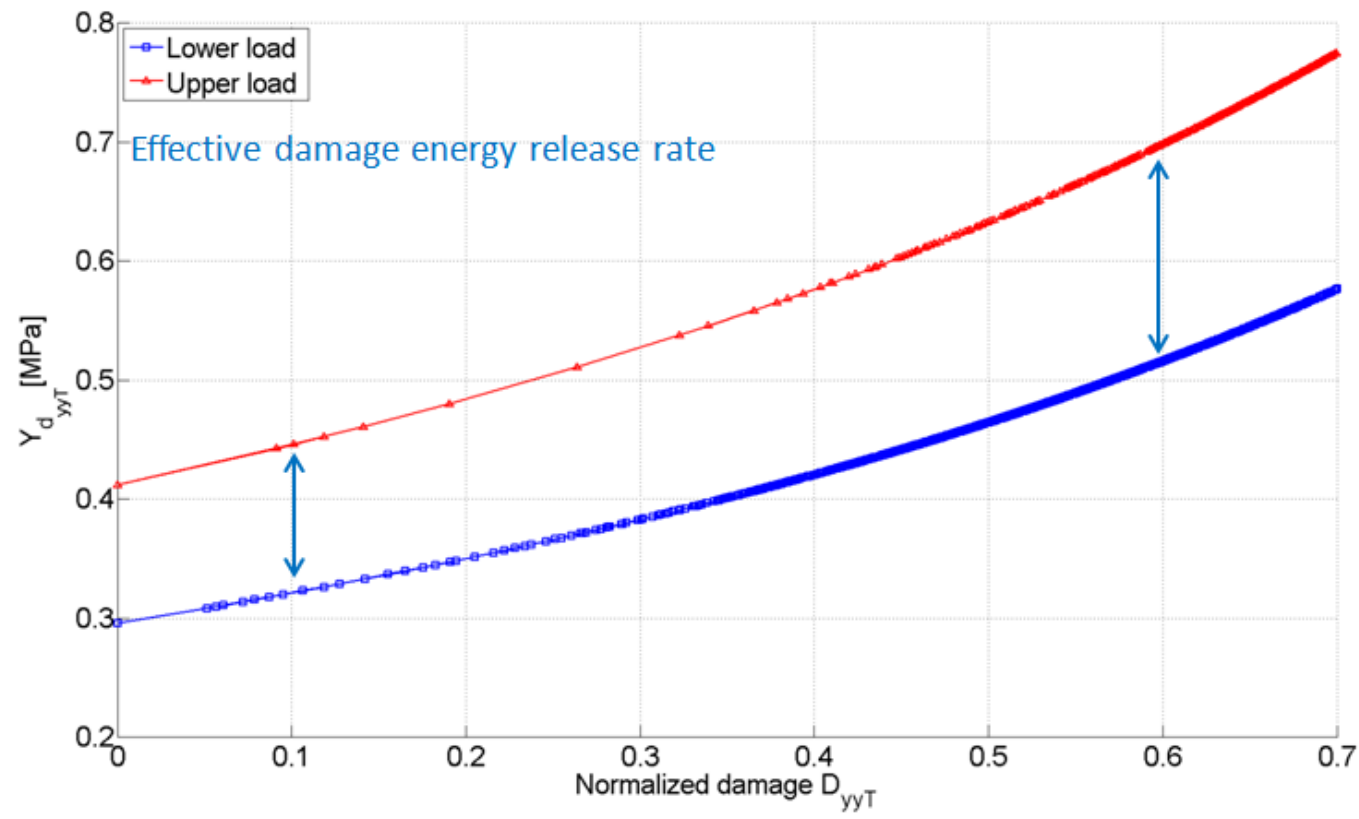

Figure 4: Displayed is the free damage energy release rate for the applied upper and lower load. The effective free damage energy release rate is marked by arrows. The amount of $Y_{\text {eff }}$ is important to calculate the effective stress. 


\section{Results and discussion}

The simulation results shown below for variable amplitude block loading with a constant stress ratio $\mathrm{R}=0.1$, for constant amplitude loading with various load ratios $\mathrm{R}$ and specimens exposed to cyclic tension - compression blocks, were solely calculated using the experimental data obtained by experiments with constant amplitude loading and a stress ratio $\mathrm{R}=0$.

Figure 5 displays the results of tests and variable amplitude block loading with a constant load ratio $R=0.1$. The purpose of this choice was to collect data for current and future studies. In the experimentally obtained stiffness-time data "steps" can be observed, which are related to the used measurement system and the change in-between two blocks with constant amplitude loading applied by the testing system.

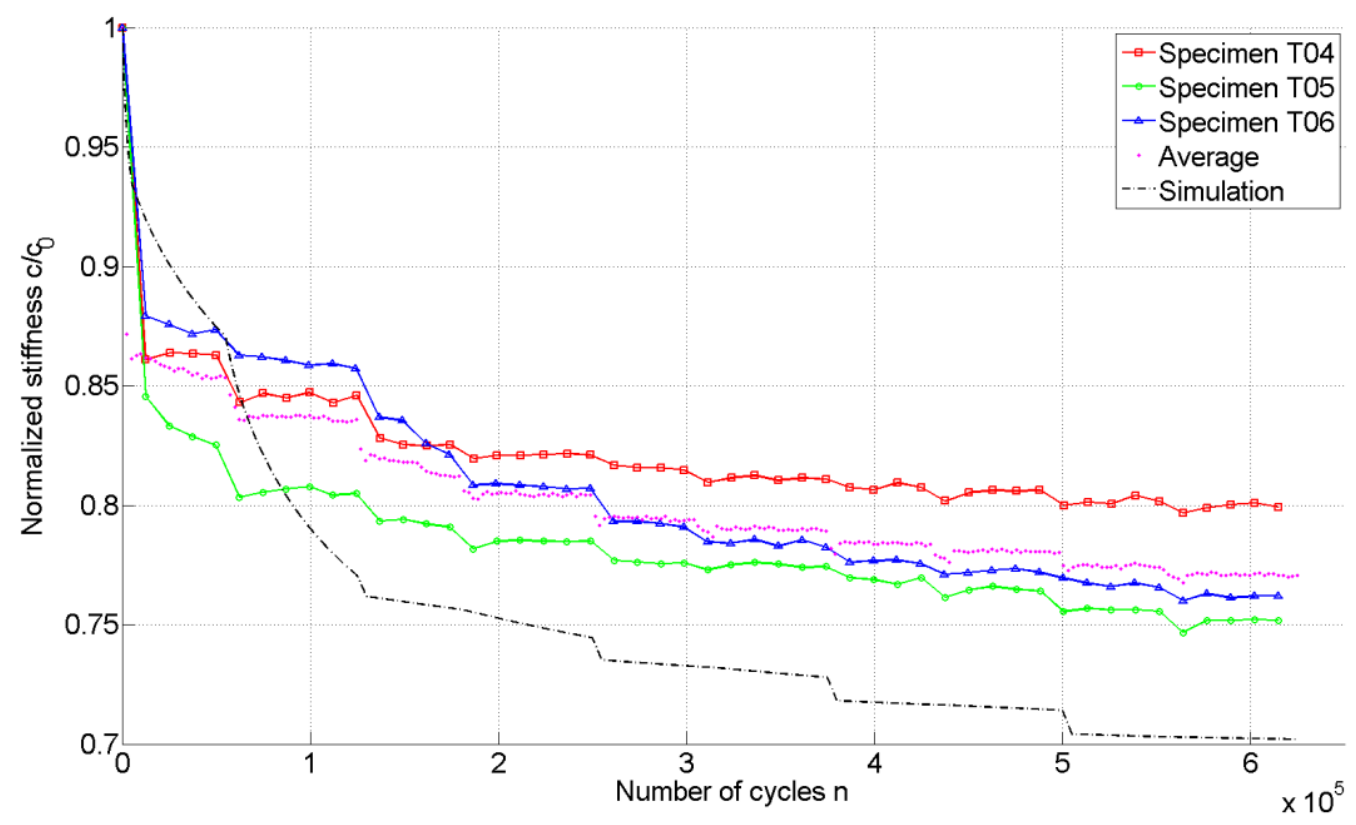

Figure 5. Comparison of data received by experiments and simulation for various amplitude block loading with a constant stress ratio of $\mathrm{R}=0.1$. Table 1 displays the applied loading block.

Figure 6 shows experimentally obtained results for constant amplitude loading with various load ratios $\mathrm{R}$. To collect data for a wide range, the load ratios were chosen as follows: $\mathrm{R}_{1}=0.1, \mathrm{R}_{2}=0.5, \mathrm{R}_{3}=0.1$ and $\mathrm{R}_{4}=0.9$. Figure 7 on the other hand shows a different material behaviour when the loading conditions are changed. The chosen load was constant as in the experiment before but the mean stress ratio $R$ was changed to $R_{1}=0.1, R_{2}=0.9$, $\mathrm{R}_{3}=0.1$ and $\mathrm{R}_{4}=0.5$. 


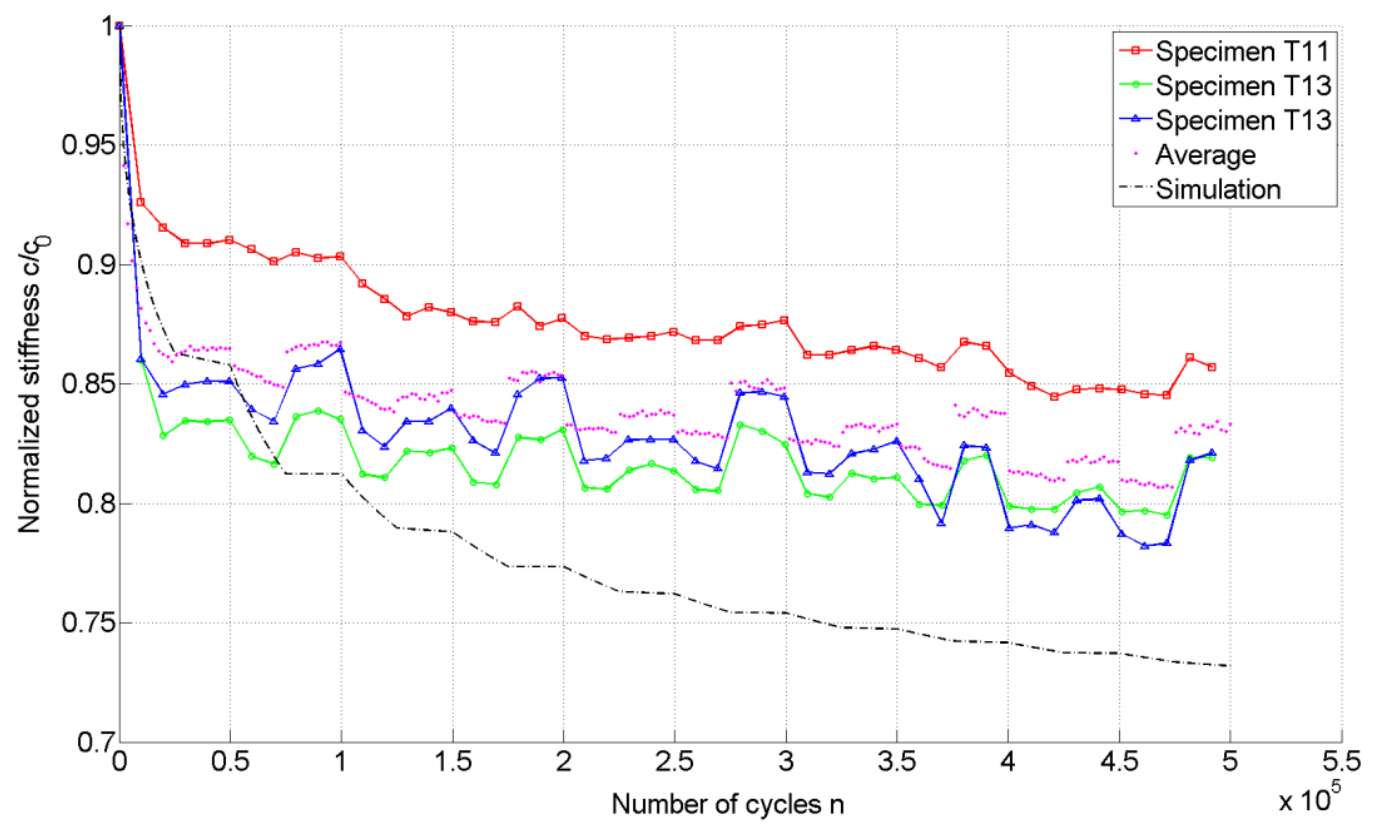

Figure 6. Comparison of experimentally received data to the numeric simulation for loading with various stress ratios $\mathrm{R}, \mathrm{R}_{1}=0.1, \mathrm{R}_{2}=0.5, \mathrm{R}_{3}=0.1, \mathrm{R}_{4}=0.9$. According to table 2 , the upper load level $\sigma_{\mathrm{UP}}$ was held constant while the lower stress level $\sigma_{\mathrm{LO}}$ was changed according to the stress ratios mentioned above.

The stiffness degradation for the loads blocks with various stress levels were calculated using the differences in the free damage energy release rate for $\sigma_{U P}$ and $\sigma_{\text {LO }}$. Having calculated the effective free damage energy release rate it is possible to calculate the effective damage.

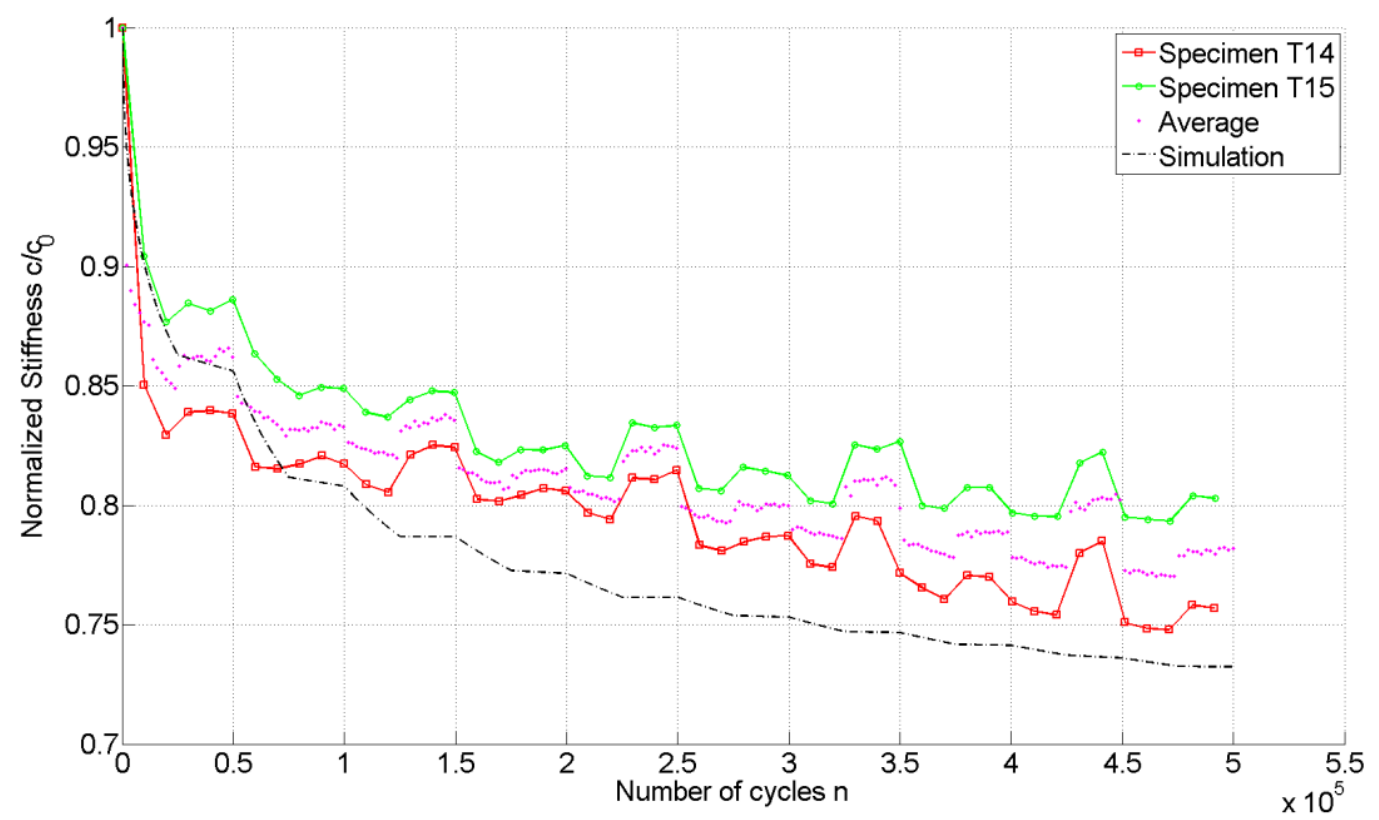

Figure 7. Comparison of experimentally received data to the numeric simulation for constant amplitude loading with various stress ratios $\mathrm{R} \mathrm{R}_{1}=0.1, \mathrm{R}_{2}=0.9, \mathrm{R}_{3}=0.1, \mathrm{R}_{4}=0.5$. The upper load level $\sigma_{\mathrm{UP}}$ was held constant while the lower stress level $\sigma_{\mathrm{LO}}$ was changed according to the stress ratios mentioned above. The applied load block can be further seen in table 3 . 
Figure 8 displays experimentally obtained results for tension compression loading blocks. The applied load was varied while the mean stress value for tension was constant with a value of $\mathrm{R}=0.1$ and for compression with $\mathrm{R}=10$. Two tension loads where applied followed by a compression and another tension load. It was planned to conduct 5 loading blocks but as the results show three out of five specimens failed before reaching the last loading block. While figure 8 displays the result of the simulation and its final failure in close proximity of the experimental obtained results. Figure 9 displays the results for the compression tension load blocks. Passive damage evolution becomes a major concern regarding cyclic loading. Material failures such as fibre kinking and buckling cause breakage of brittle fibres which do not transmit compressive stresses after load reversals. Although the simulation was tested with and without a passive damage criterion for cycling loading described by Matzenmiller [17] no better results regarding the number of cycles could be obtained.

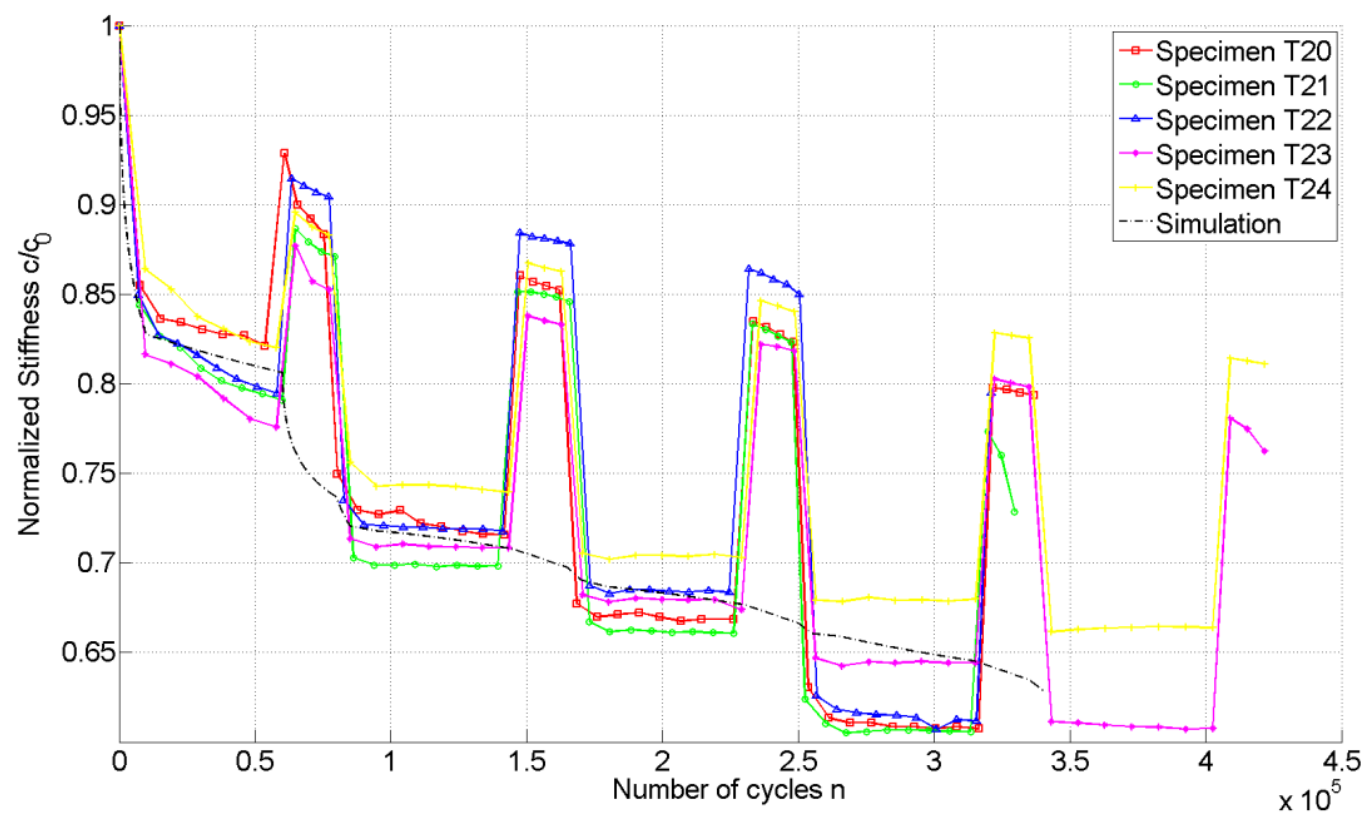

Figure 8. Comparison of experimentally received data to the numeric simulation for various loading blocks. While the loading ratio is kept constant $\mathrm{R}=0.1$ for tension and $\mathrm{R}=10$ for compression the applied loads were varied (table 4).

Two compression loads were followed by one tension and another compression loading block. While the results in figure 8 are quite accurate the simulation results of figure 9 are lacking in accuracy not only in stiffness but also in the obtained number of cycles to failure. While the difference in stiffness can be explained by the use of only one modulus (for tension and compression) the number of cycles must be looked at more carefully. 


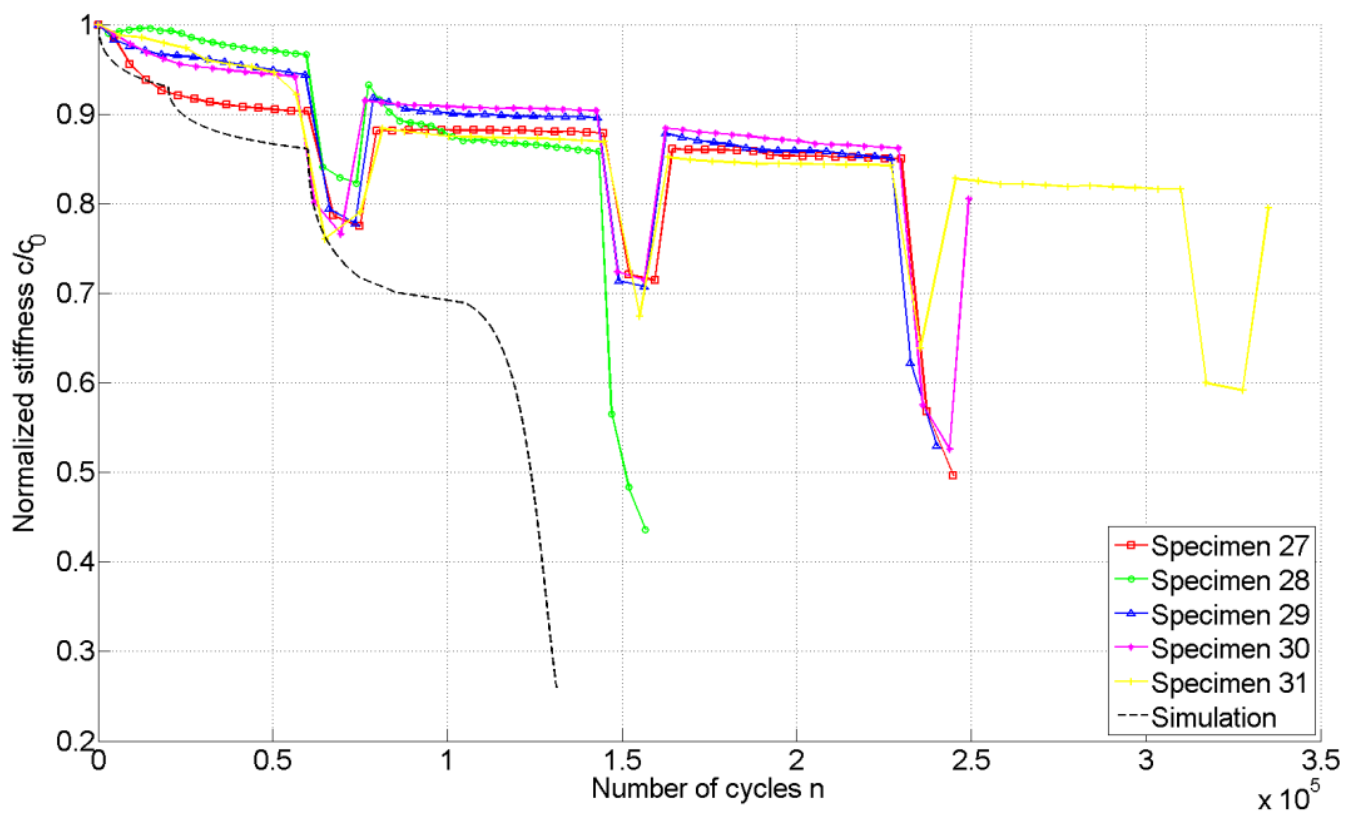

Figure 9. Comparison of experimentally received data to the numeric simulation for various load blocks. While the loading ratio is kept constant $\mathrm{R}=0.1$ for tension and $\mathrm{R}=10$ for compression the applied loads were varied (table 5).

\section{Conclusion and summary}

This contribution presents experimentally and numerically obtained fatigue data of carbon fibre reinforced multidirectional laminates, produced with high pressure resin transfer moulding. Variable amplitude block loading with constant and variable load ratio $\mathrm{R}$ as well as under constant amplitude loading with various load ratios $\mathrm{R}$ was applied. The material parameters for the numerical simulation were obtained from test coupons using constant amplitude loading $(\mathrm{R}=0)$. The data were further used to calculate the damage energy release rate. Using equations 3,4 and 5 , it was possible to simulate the material behaviour under variable amplitude block loading. In most cases, a good correlation between simulation and experiment could be obtained.

Further studies need to be conducted to explain the correlation of tension and compression damage. Currently this problem is solved in a quite robust way using damage factors for cyclic tension and for cyclic compression loads. Ideally, this issue should be solved relating a part of the produced damage energy to compression and vice versa. Therefore compression studies of micromechanical damage initiation for tension and compression should be conducted. This approach should also be able to solve the problem of predicting stiffness degradation and final failure and rupture of the sample for load conditions with for general variable amplitude loading.

Additionally experiments are conducted to validate the proposed method for unidirectional materials. Furthermore it should be possible to predict the degradation behaviour of the tested multidirectional laminates using only the damage energy release rate of the unidirectional materials. If successful it will be possible to predict the fatigue behaviour of the tested multidirectional laminates and therefore simplify the testing and simulation procedure. 


\section{REFERENCES}

[1] R. Talreja and Chandra Veer Singh. Damage and failure of composite materials. Cambridge University Press, Cambridge, 2012. ISBN 978-1-280-77369-3.

[2] W. Chan. Composites Engineering Handbook. New York, Marcel Dekker Inc., pp. 309-370. Marcel Dekker Inc., 1997. ISBN 0824793048.

[3] J. Degrieck and W. Van Paepegem. Fatigue damage modelling of _bre-reinforced composite materials: Review. Applied Mechanics Reviews, 54(4):279 - 300, 2001.

[4] B. Harris. Fatigue in Composites.

[5] I.M. Daniel, O. Ishai, Engineering Mechanics of Composite Materials, Oxford University Press, 2006 (second edition).

[6] R. Talreja, C. V. Singh, Damage and Failure of Composite Materials, Cambridge Univerity Press, 2012.

[7] P. Ladevèze, E. Le Dantec, Damage Modelling of elementary ply for laminated composites, Composites Science and Technology 43 (1992), pp. 257-267.

[8] G. Pinter, E. Ladstätter, W. Billinger, R. Lang, Characterisation of the tensile fatigue behaviour of RTM-laminates by isocyclic stress-strain-diagrams, International Journal of fatigue 28 (2006),pp.1277-1283.

[9] P. Ladevèze, A Damage computational method for composite structures, Computers \& Structures Vol. 77 (1192) pp. 79-87.

[10] O. Allix, Modelling and identification of the mechanical behaviour of composite laminates in compression, Composites Science and Technology 51 (1994), pp. 35-42.

[11] P. Ladevèze and E. LeDantec. Damage modelling of the elementary ply for laminated composites. Composites Science and Technology, 43(3):257 - 267, 1992. ISSN 0266-3538.

[12] P. Ladevèze. An anisotropic damage theory with unilateral effects. In Oliver Allix and Francis Hild, editors, Continium Damage Mechanics of Materials and Structures, chapter Chapter 2: Mechanisms-Related Continuum Damage Mechanics. Elsevier, 2002. ISBN 0-08-043918-7.

[13] D. G. Degeroges and P. Ladevèze. Course on Emerging Techniques for Damage Prediction and Failure Analysis of Laminated Composite Structures. Cépaduès-Editions, 2008.

[14] G. Lubineau. A pyramidal modeling scheme for laminates - identification of transverse cracking. International Journal of Damage Mechanics, 19(4):499 - 518, May 2010. ISSN 1056-7895.

[15] P. Paris and F. Erdogan, "A critical analysis of crack propagation laws", Journal of Basic Engineering, Transactions of the American Society of Mechanical Engineers, December 1963, pp.528-534.

[16] W. Van Paepegem and J. Degrieck. A new coupled approach of residual stiffness and strength for fatigue of fibre-reinforced composites. International Journal of Fatigue, 24(7):747 - 762, 2002. ISSN 0142-1123.

[17] A. Matzenmiller, J. Lubliner, R.L. Taylor, A constitutive model for anisotropic damage in fiber-composites, Mechanics of Materials 20 (1995) 125-152. 